\title{
Metabolic Response to Four Weeks of Muscular Endurance Resistance Training
}

John W. Farrell III ${ }^{*}$, David J. Lantis ${ }^{1}$, Carl J. Ade ${ }^{2}$, Debra A. Bemben ${ }^{1}$, Rebecca D. Larson ${ }^{1}$

${ }^{1}$ Department of Health and Exercise Science, University of Oklahoma, 1401 Asp Ave, Norman, OK, 73019, USA, ${ }^{2}$ Department of Kinesiology, Kansas State University, 920 Denison Ave, Manhattan, KS, 66506, USA

Corresponding Author: John W. Farrell III, E-mail: John.W.Farrell-1@ou.edu

\section{ARTICLE INFO}

Article history

Received: July 25, 2017

Accepted: September 27, 2017

Published: October 31, 2017

Volume: 5 Issue: 4

Conflicts of interest: None

Funding: None

\begin{abstract}
Background: Previous investigations have shown that muscular endurance resistance training (MERT) is conducive in improving the onset of blood lactate accumulation (OBLA). However, the metabolic response and time course for adaption is still unclear. Objective: The aims of the current study were to evaluate and track the metabolic response to an individual session of MERT as well as to assess performance adaptations of supplementing an aerobic exercise training program with four weeks of MERT. Methods: Seventeen aerobically active men were randomly assigned to either the experimental (EX) or control group $(\mathrm{CON}), 9 \mathrm{EX}$ and $8 \mathrm{CON}$. Baseline measures included a graded exercise test (GXT) and 1-repetition maximum (1RM) testing for leg press (LP), leg curl (LC), and leg extension (LE). CON continued their regular aerobic activity while the EX supplemented their regular aerobic exercise with 4 weeks of MERT. Results: No significant group differences were observed for all pre-training variables. Following four weeks of training no significant differences in cardiorespiratory or metabolic variables were observed for either group. However, significant improvements in LC and LE 1-RM were observed in EX compared to CON. Substantial accumulations in blood lactate were observed following each MERT session. Conclusion: Four weeks of MERT did not improve cardiorespiratory or metabolic variables, but did significantly improve LC and LE. MERT was also observed to induce a blood lactate response similar to that of HIIT. These findings suggest greater than four weeks is need to see metabolic adaptations conducive for improved aerobic performance using MERT.
\end{abstract}

Key words: Oxygen Consumption, Physical Endurance, Resistance Training, Lactates, Monocarboxylic Acid Transporters

\section{INTRODUCTION}

The development of optimal training methods has been an area of great interest for coaches, athletes and scientists. Current research suggests that blood lactate performance curves provide both reliable and valid information for prescribing exercise intensity due to the strong relationship between various lactate thresholds and performance. Several studies have established a strong correlation between the onset of blood lactate accumulation (OBLA) and aerobic exercise performance (Bergman et al., 1999; Brooks, 2010; Figueira, Caputo, Pelarigo, \& Denadai, 2008; Laursen \& Jenkins, 2002; Rogatzki, Wright, Mikat, \& Brice, 2014; Tanaka \& Swensen, 1998). OBLA has been defined as the intensity at which blood lactate reaches a fixed number that is equal to a concentration of $4 \mathrm{mmol} / \mathrm{L}$ (Figueira et al., 2008; B Sjödin \& Jacobs, 1981), and corresponds to the point at which exercise intensity transitions from tolerable to sever (Figueira et al., 2008). Therefore aerobic performance can be improved if the intensity at which OBLA occurs shifts to a higher value, for example running speed, cycling workload, or $\mathrm{VO}_{2}$.

Recent studies have established a new method for the improvement of OBLA utilizing resistance training with lighter loads and higher repetitions, termed muscular endurance resistance training (MERT). Previous studies have demonstrated that a single bout of resistance training with lighter loads and higher repetitions can induce large accumulations of lactate and a simultaneous drop in $\mathrm{pH}$ (Edge, Hill-Haas, Goodman, \& Bishop, 2006; Rogatzki et al., 2014; Schott, McCully, \& Rutherford, 1995) that are comparable to values seen with high intensity interval training sessions (HIIT) (Edge, Bishop, \& Goodman, 2006; Edge, Hill-Haas, et al., 2006), and greater than that of a traditional strength training session (Kraemer et al., 2002; Rogatzki et al., 2014). Researchers have observed improvements in watts and $\mathrm{VO}_{2}$ at which OBLA occurs during cycling following 8 weeks of MERT (Farrell III, Lantis, Ade, Cantrell, \& Larson, 2017; Lantis, Farrell III, Cantrell, \& Larson, 2017)). Rightward shifts in blood lactate curves translate into athletes exercising at a 
higher workload or higher percentage of their $\mathrm{VO}_{2}$ max before experiencing the negative side effects of lactate accumulation. Researchers have speculated that the improvements in lactate kinetics are due to improvements in the metabolic clearance rate of lactate through increases in monocarboxyl transfer proteins (Cruz et al., 2012). Researchers have proposed that the improvements in lactate kinetics observed with HIIT could potentially be the same in MERT. However, the lactate response to several training session throughout a MERT program is unclear. It is not known if the response will decrease over time indicating improvements in lactate kinetics or will continue to be elevated throughout the duration of the program. The tracking of the lactate response could also give insight into the relative metabolic intensity that athletes are training at during each session.

With MERT just recently being utilized to develop improvements in OBLA, proper programming and optimization of this method still needs to be established. Previous studies have reported improvements in OBLA using 8 weeks of muscular endurance training with 1 minute of rest between sets of exercise ((Farrell III et al., 2017; Lantis et al., 2017). However, improvements in OBLA and aerobic power following HIIT and Sprint Interval Training (SIT) have been seen in as little as 4 to 8 sessions over 2 to 4 weeks (Beyranvand, 2017; Burgomaster, Heigenhauser, \& Gibala, 2006; Burgomaster, Hughes, Heigenhauser, Bradwell, \& Gibala, 2005; Jakeman, Adamson, \& Babraj, 2012; Laursen, Shing, Peake, Coombes, \& Jenkins, 2005; B. Rønnestad, Hansen, \& Ellefsen, 2014; Talanian, Galloway, Heigenhauser, Bonen, $\&$ Spriet, 2007). With the proposed mechanisms of adaptations between MERT and HIIT being the same, it can be speculated that similar results can be observed over a similar length of time. The optimal length of rest between sets of exercise is still unclear as well.

Therefore, the purpose of the current study was to examine the cardiorespiratory and metabolic adaptations obtained from the supplementation of an aerobic training program with a 4 week MERT utilizing 30 second rest interval between sets of exercise. Additionally, the purpose of the current study was to evaluate and track the blood lactate response to individual training sessions throughout the course of the training program. It was hypothesized that an aerobic training program supplemented with 4 weeks of MERT with 30 second rest intervals would result in greater improvements in the OBLA and gas exchange threshold (GET) when compared to aerobic training alone. It was also hypothesized that there would be no significant improvements in maximal oxygen uptake $\left(\mathrm{VO}_{2} \max \right)$ when compared to aerobic training alone.

\section{METHOD}

\section{Subjects}

Nineteen male participants (18-37 years old) were recruited for the current study. 17 participants completed the study with 2 participants dropping out due to time commitment issues. $\mathrm{G}^{*}$ Power software (version 3.1.9.2) $(\alpha$ level $=0.05$ and effect size $=0.08$ ) was used to determine proper sample size. Subjects were randomly divided into an experimental (EX) group, whose regular aerobic training was supplemented with a muscular endurance training program, or a control $(\mathrm{CON})$ group who continued their regular aerobic exercise training without the addition of muscular endurance training. All subjects were considered to be aerobically active and had not participated in any resistance training for 6 months prior to start of the study; determined with a self-reported physical activity questionnaire. Aerobically active was defined as having participated in aerobic exercise at least one hour per day for 3 days per week for the past 6 months. All subjects were required to reach a $\mathrm{VO}_{2}$ max of at least $40 \mathrm{ml} / \mathrm{kg}$ / min to participate in the study. This threshold has been used previously in similar studies (Farrell III et al., 2017; Lantis et al., 2017), and it indicates an aerobic capacity of good/ excellent (Heyward, 1992). This study was approved by the Institutional Review Board at the University of Oklahoma, and each subject gave a verbal and written informed consent before participation. All testing and training was completed in an air-conditioned laboratory at a temperature $20-25^{\circ} \mathrm{C}$.

\section{Experimental Protocol}

Prior to and following the 4 week training period both groups performed a staged graded exercise test to determine OBLA using the fixed at 4mmol/L method(Bertil Sjödin, Jacobs, \& Svedenhag, 1982), GET and, $\mathrm{VO}_{2} \max$. Maximal strength for leg press (LP), leg curl (LC), and Leg extension (LE) was assessed using 1-repetition maximum (1-RM) All subjects were instructed to continue their low-intensity aerobic training without making alterations to volume or intensity. In addition to their current low-intensity aerobic training the EX group returned to the laboratory two times per week for four weeks to perform muscular endurance training under the supervision of the researchers.

\section{Incremental exercise test}

A magnetically braked cycle ergometer (Sport Excalibur, Lode; B.V. Medical Technology, Groningen, The Netherlands) along with a metabolic cart (True One 2400, Parvo Medics, Sandy, UT) was utilized to perform an incremental exercise test to determine $\mathrm{VO}_{2}$ max, GET, and OBLA. It was chosen to allow for easy attainment of plasma lactate measurements. Subjects were instructed to abstain from exercise and caffeine twelve hours prior to testing and to fast three to four hours prior to testing. A urine sample was obtained to determine urine specific gravity using a refractometer (model CLX-1, VEE GEE Scientific Inc., Kirkland, WA). Subjects had to have a urine specific gravity between 1.004 and 1.026 to be considered adequately hydrated to perform the incremental exercise test. In the instance a participant was not adequately hydrated they were instructed to consume a glass of water and rest for 30 minutes before collecting a second sample. If at that time they were still under hydrated they were rescheduled for a subsequent day. A resting fingertip capillary blood sample was collected to determine whole blood lactate concentration prior to testing using a commercial lactate meter (Lactate Plus, Nova Biomedical, Waltham, 
MA) that was calibrated with known lactate standards (Lactate Plus, Lac Control Level 1, 1.0-1.6 mM) (Lactate Plus, Lac Control Level 2, 4.0-5.4 mM) before each use. Following a one minute rest period and a five minute warm up at 50 watts $(\mathrm{W})$, the staged exercise test was initiated at a work rate of $125 \mathrm{~W}$ and increased by $25 \mathrm{~W}$ every three minutes until the participant reached their limit of exercise tolerance indicated by a pedal rate dropping below 50 revolutions per minute. At the end of each of the three-minute stage blood lactate and rating of perceived exertion (RPE) based on the Borg Scale (Borg, 1970) was measured. The $\mathrm{VO}_{2}$ and work rate $(\mathrm{W})$ corresponding to $4.0 \mathrm{mmol} / \mathrm{L}$ (OBLA) was calculated by plotting $\mathrm{VO}_{2}$ and $\mathrm{W}$ against blood lactate concentration and using linear interpolation. Metabolic and ventilatory data were continuously measured and averaged over $30 \mathrm{sec}-$ ond intervals. The work rate corresponding to the GET was determined by graphing: 1) $\mathrm{VCO}_{2}$ as a function of $\left.\mathrm{VO}_{2}, 2\right)$ the factional concentration of end-tidal of $\mathrm{O}_{2}$ and $\mathrm{CO}_{2}\left(\mathrm{FETO}_{2}\right.$ and $\mathrm{FETCO}_{2}, 3$ ) the ventilatory equivalents for $\mathrm{O}_{2}$ and $\mathrm{CO}_{2}$ $\left(\mathrm{VE} / \mathrm{VO}\right.$, and $\left.\mathrm{VE} / \mathrm{VCO}_{2}\right)$, and 4) respiratory exchange ratio (RER; $\mathrm{VCO}_{2} / \mathrm{VO}_{2}$ ). The vertical alignment of these profiles allows for the verification of the GET via the work rate that aligned with: 1) an increase in $\mathrm{VCO}_{2}$ out of proportion to $\mathrm{VO}_{2}, 2$ ) an increase in $\mathrm{VE} / \mathrm{VO}_{2}$ and no increase in $\mathrm{VE} / \mathrm{VCO}_{2}$, $3)$ a rise in $\mathrm{FETCO}_{2}$ and $\mathrm{FETO}_{2}$, and 4) no inflection of the RER (can be a result from changes in $\mathrm{CO}_{2}$ storage dynamics unrelated to blood lactate buffering) (Beaver, Wasserman, \& Whipp, 1986).

\section{Repetition maximum (1-RM) testing}

1-RM testing was performed to assess maximum strength for leg press (LP), leg curl (LC) and leg extension (LE) based on recommendations of the National Strength and Conditioning Association (Baechle \& Earle, 2008). In brief, several sub-maximal repetitions were performed to serve as a warmup. An initial weight was selected to be within 50 to 70 percent of the participant's perceived capacity. The weight was increased incrementally until a weight that could be lifted once but not twice was achieved. Three minutes of rest was given between each attempt. If a participant was able to lift the entire weight stack they were required to complete a full range of motion and immediately attempt additional repetitions through a full range of motion until failure. The number of additional repetitions was then used to calculate their 1-RM (Baechle \& Earle, 2008).

\section{Aerobic exercise}

Based on previous studies (Farrell III et al., 2017; Lantis et al., 2017) (Karsten, Stevens, Colpus, Larumbe-Zabala, \& Naclerio, 2016), subjects were instructed to maintain current aerobic training volume, and intensity throughout the study. If subjects indicated a decrease of $>1 / 3$ or an increase of $>5 \%$ in training volume subjects were excluded from the study in order to prevent detraining and additional aerobic adaptations (Baechle \& Earle, 2008; Fleck, 1994; Zupan \& Petosa, 1995). Also any indications in an increase in aerobic training intensity resulted in exclusion from the study.

\section{Muscular endurance resistance training}

The training program consisted of supervised sessions on 2 days* wk $^{-1}$ over a four week period to supplement participants aerobic training program. Participants were instructed to not perform any resistance training outside of the study. Every training session began with the subject arriving to the lab and resting for 10 minutes before a resting blood lactate measurement was taken using a commercial lactate meter (Lactate Plus, Nova Biomedical, Waltham, MA). Each resistance training session included a warmup consisting of 5 to 10 repetitions at 30 to 40 percent of their 1RM followed by four sets of 12 to 15 repetitions at $50 \%$ of the subject's established 1-RM for LP, LC, and LE (Cybex Strength Systems) with 30 seconds of rest between sets and 3 minutes of rest between exercises. Immediately after the completion of the final set of exercise a post-training lactate sample was taken. After 2 weeks (4 training sessions) the training weight was increased by 4.54 kilograms (a 2 to $4 \%$ increase) if an individual was able to complete four sets of 15 repetitions to compensate for any strength gains (Baechle \& Earle, 2008). If participants were unable to complete four sets of 15 repetitions the training weight was kept the same.

\section{Statistical analysis}

Data are presented as means \pm SD. Delta scores (post-pre) are indicated by $\Delta$. Delta scores were used for data analysis to negate any differences noted in pre measurements due to both groups consisting of individuals that participated in several different aerobic activities (cycling, running, and triathlon). In addition the incremental exercise test was not sport specific for all subjects. Independent t-tests were utilized to determine if significant between group differences existed between the EX and CON groups. Repeated measures ANOVA was utilized to determine if significant differences existed in lactate levels between training visits. All statistical analyses were performed using SigmaPlot (Version 12.5, Systat Software Inc., San Jose, CA). Cohen's d effect sizes (ES) were reported for all significant measures. A value of $\leq 0.20$ was considered a weak effect, a value of $\leq 0.50$ was considered a moderate effect, and a value of $\geq 0.80$ was considered a strong effect (Cohen, 1988). An alpha level of $\mathrm{p} \leq 0.05$ was set for the level of significance.

\section{RESULTS}

\section{Subject Characteristics and Training Compliance}

Descriptive characteristics are summarized in Table 1. No statistical differences $(p>0.05)$ existed between groups for age, height, and body mass. Of the 9 subjects included in the EX group all participants completed at least 12 repetitions per set for all training sessions. All subjects met the requirements for increasing training load after the $4^{\text {th }}$ training session.

\section{Incremental Exercise Test}

The physiological measurements from the incremental exercise test are summarized in Table 2. No significant differ- 
ences $(p>0.05)$ were observed for any of the physiological variables following the study intervention period between groups.

\section{Maximal Strength Assessment}

1-RM strength values are summarized in Table 3. Following the study intervention significant group differences existed for $\mathrm{LC}(\mathrm{p}=0.002 ; \mathrm{ES}=1.79)$ and $\mathrm{LE}(\mathrm{p}=0.028 ; \mathrm{ES}=1.20)$ between the EX and CON groups.

\section{Training Session Blood Lactate Response}

The average blood lactate response following each MERT session for the EXP group is illustrated in Figure 1. No statistically significant differences were observed in blood lactate levels between training sessions $(\mathrm{p}>0.05)$. The average individual blood lactate response across all MERT sessions for the EXP group is summarized in Figure 2.

\section{DISCUSSION}

The purposes of the current study were: (1) to examine the physiological and metabolic adaptions from the supplementation of an aerobic training program with 4 weeks of MERT, (2) to evaluate the effects of 30 second rest periods between

Table 1. Subject characteristics (Mean $\pm(\mathrm{SD}))$

\begin{tabular}{lccccc}
\hline Variables & \multicolumn{4}{c}{ Groups } \\
\cline { 2 - 3 } & \multicolumn{1}{c}{ Experimental (n=9) } & & \multicolumn{2}{c}{ Control (n=8) } \\
\cline { 2 - 3 } \cline { 5 - 6 } & Pre & Post & & Pre & Post \\
\hline Age (years) & $21.4 \pm 2.1$ & $21.6 \pm 2.1$ & & $25.5 \pm 6.4$ & $25.6 \pm 6.3$ \\
Height (cm) & $180.2 \pm 5.3$ & $180.2 \pm 5.3$ & & $179.5 \pm 3.9$ & $179.5 \pm 3.8$ \\
Body & $75.2 \pm 10.0$ & $75.2 \pm 8.6$ & & $77.2 \pm 11.8$ & $76.6 \pm 11.4$ \\
mass (kg) & & & & \\
\hline
\end{tabular}

Differences if present were denoted using $*(p<0.05)$. Standard deviations represent variability sets of MERT, and (3) monitor and track blood lactate responses to MERT sessions. In agreement with the researchers' hypothesis the addition of the MERT did not improve $\mathrm{VO}_{2} \max$. In contrast to the researchers' hypothesis, the addition of the MERT did not improve OBLA or GET.

A large majority of previous studies that have investigated the effects of resistance training on endurance performance have utilized a traditional strength training protocol (high intensity low repetition). Several of these studies indicated improvements in various endurance performance measures but overwhelming majority have reported no improvements in $\mathrm{VO}_{2}$ max when using aerobically trained subjects (Aagaard et al., 2011; Jung, 2003; Losnegard et al., 2011; Mikkola et al., 2011; Millet, Jaouen, Borrani, \& Candau, 2002; B. R. Rønnestad, Hansen, \& Raastad, 2010; B. R. Rønnestad, Kojedal, Losnegard, Kvamme, \& Raastad, 2012). Previous studies that have used a lower intensity higher repetition resistance training program saw similar results. Edge et al.(2006). found no improvements in $\mathrm{VO}_{2} \max$ following a high repetition resistance training program for 5 weeks in recreationally active females (Edge, Hill-Haas, et al., 2006). Campos et al. (2002) observed no improvements in $\mathrm{VO}_{2} \mathrm{~m}-$ ax in untrained males following 8 weeks of high repetition resistance training (20 to 28 reps) training (Campos et al., 2002). Both Lantis et al. (2017). and Farrell et al. (2017) used identical MERT protocols and saw no improvements in $\mathrm{VO}_{2} \max$ (Farrell III et al., 2017; Lantis et al., 2017). The findings of the current study in regards to no improvements in VO2max are in agreement with previous studies. The current study also observed no significant improvements in GET or $\mathrm{W}$ at GET following the training intervention. This finding is in agreement with Farrell et al. (2017) who also observed no improvements in GET or W at GET (Farrell III et al., 2017). It can be speculated that even with 30 second rest intervals MERT does not provide an efficient cardiorespiratory stimulus to see improvements in $\mathrm{VO}_{2} \max$ or GET. Although no cardiorespiratory adaptations $\left(\mathrm{VO}_{2} \max\right.$ or GET) have been linked with MERT, metabolic or local muscular

Table 2. Physiological data during incremental exercise test (Mean \pm SD)

\begin{tabular}{|c|c|c|c|}
\hline \multirow[t]{3}{*}{ Variable } & \multicolumn{2}{|c|}{ Groups } & \multirow[t]{3}{*}{ Effect size } \\
\hline & Experimental $(n=9)$ & Control $(n=8)$ & \\
\hline & $\Delta$ & $\Delta$ & \\
\hline $\mathrm{VO}_{2} \mathrm{Abs}(1 / \mathrm{min})$ & $0.019 \pm 0.173$ & $0.077 \pm 0.139$ & 0.369 \\
\hline $\mathrm{VO}_{2} \operatorname{Rel}(\mathrm{ml} / \mathrm{kg} / \mathrm{min})$ & $0.644 \pm 2.91$ & $1.06 \pm 2.31$ & 0.158 \\
\hline $\operatorname{GET}(1 / \mathrm{min})$ & $0.10 \pm 0.252$ & $0.029 \pm 0.150$ & 0.342 \\
\hline Watts@GET & $10.7 \pm 19.7$ & $7.14 \pm 12.20$ & 0.218 \\
\hline $\mathrm{VO}_{2}(\mathrm{~L} / \mathrm{min}) @ \mathrm{OBLA}$ & $0.0 \pm 0.357$ & $0.100 \pm 0.141$ & 0.368 \\
\hline $\mathrm{VO}_{2}(\mathrm{ml} / \mathrm{kg} / \mathrm{min}) @$ OBLA & $0.031 \pm 4.67$ & $1.72 \pm 2.09$ & 0.467 \\
\hline Watts@ OBLA & $-7.24 \pm 12.09$ & $3.54 \pm 9.21$ & 1.00 \\
\hline$\%$ of $\mathrm{VO}_{2}$ max OBLA achieved & $-1.01 \pm 6.70$ & $2.07 \pm 3.15$ & 0.588 \\
\hline Peak Power $(\mathrm{W})$ & $8.33 \pm 17.68$ & $-3.13 \pm 8.84$ & 0.820 \\
\hline
\end{tabular}

$\Delta$ denotes gain scores (post - pre). Differences between groups were denoted using $*(p<0.05)$. Standard deviations represent variability. $\mathrm{VO}_{2}$ Abs: Absolute $\mathrm{VO}_{2}$; $\mathrm{VO}_{2}$ Rel: Relative $\mathrm{VO}_{2}$; GET: Gas Exchange Threshold; OBLA: Onset of Blood Lactate Accumulation at $4 \mathrm{mmol} / \mathrm{L}$ of lactate. 
Table 3. 1-RM measurements (Mean \pm SD)

\begin{tabular}{|c|c|c|c|}
\hline \multirow[t]{3}{*}{ Variable } & \multicolumn{2}{|c|}{ Groups } & \multirow{3}{*}{$\begin{array}{c}\text { Effect } \\
\text { size }\end{array}$} \\
\hline & $\begin{array}{c}\text { Experimental } \\
(\mathbf{n}=9)\end{array}$ & $\begin{array}{c}\text { Control } \\
(n=8)\end{array}$ & \\
\hline & $\Delta$ & $\Delta$ & \\
\hline Leg press (kg) & $20.5 \pm 34.5$ & $1.99 \pm 6.58$ & 0.745 \\
\hline Leg Curl (kg) & $9.21 \pm 5.35^{*}$ & $-0.142 \pm 5.08$ & 1.79 \\
\hline Leg extension $(\mathrm{kg})$ & $9.93 \pm 9.43 *$ & $1.00 \pm 4.67$ & 1.20 \\
\hline
\end{tabular}

$\Delta$ denotes gain scores (post - pre). Differences between groups were denoted using $*(p<.05)$. Standard deviations represent variability

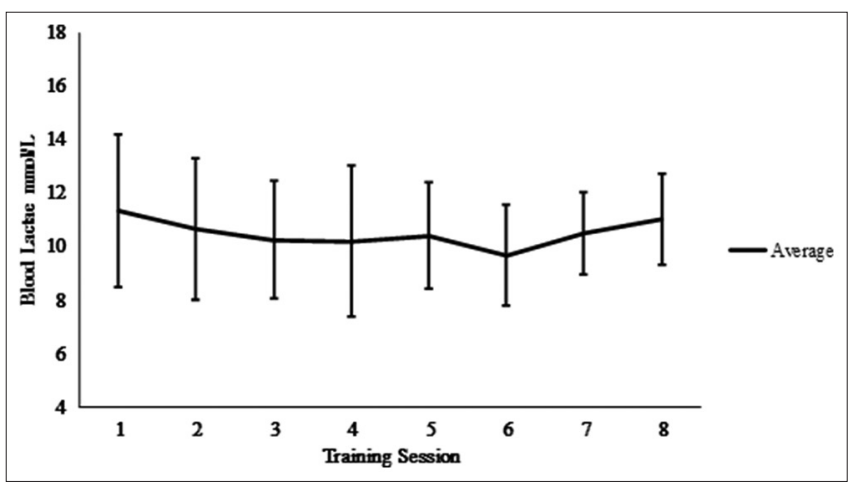

Figure 1. Average blood lactate response (post-pre) to each MERT

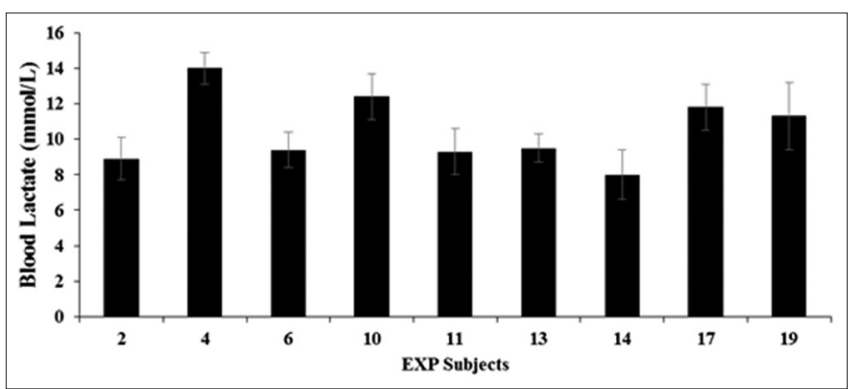

Figure 2. Average individual blood lactate response across all sessions of MERT

adaptations have resulted in improvements in OBLA. Farrell et al. (2017) observed a significant improvement in the $\mathrm{VO}_{2}$ in which OBLA occurred following MERT (Farrell III et al., 2017). As a result, OBLA was achieved at a $\mathrm{VO}_{2} 7.2 \%$ closer to $\mathrm{VO}_{2} \max$ following training. Lantis et al. (2017) showed an increase in watts (W) at which OBLA occurred on a cycle ergometer (Lantis et al., 2017). It was speculated that the rightward shift in OBLA observed after 8 weeks of MERT in both studies was due to improvements in the metabolic clearance rate of lactate via increases in MCT proteins (Cruz et al., 2012; Donovan \& Brooks, 1983). However, no improvements in lactate kinetics were observed in the current study. The current researchers have speculated that the reduced number of MERT sessions, compared to previous studies, did not allow for proper metabolic adaptations.

Following the MERT intervention the EXP group showed a significant increase in 1RM strength for both LC and LE. This provides support that the program was effective in eliciting adaptations conducive for improvements in 1RM strength. Due to the lack of resistance training in the subjects' training history and the length of the MERT intervention it can be speculated that the improvements in 1RM strength observed were due to improvements in neural recruitment (Baechle \& Earle, 2008). These findings are in agreement with previous research on MERT (Farrell III et al., 2017; Lantis et al., 2017).

Previous studies have shown that resistance training utilizing higher repetitions with lower resistance induces a large accumulation in lactate and a simultaneous drop in $\mathrm{pH}$ similar to that observed during HIIT (Edge, Bishop, et al., 2006; Edge, Hill-Haas, et al., 2006; Rogatzki et al., 2014; Schott et al., 1995). Previous investigations examining MERT have speculated that the training protocol also induces a similar lactate response observed with HIIT. However, previous investigations with MERT have not actually measured lactate responses. In the current study, prior to and immediately after each MERT session a blood sample was taken to assess blood lactate concentrations. The average blood lactate response (post-session - pre-session) for each training session is shown in Figure 1. The current study showed an average lactate response of $10.5 \pm 1.9 \mathrm{mmol} / \mathrm{L}$ to the MERT, indicating a similar lactate response to that of HIIT (Edge, Bishop, et al., 2006). When examining the individual responses to the same relative workload a wide range of responses were observed. Lactate responses for the first four MERT sessions ranged from $7.0 \pm 0.716$ to $13.8 \pm 1.76$ while the lactate response to the final four MERT sessions (following the increase in training load) ranged from $8.23 \pm$ 0.956 to $13.8 \pm 0.763$. This wide range of responses shows that although all subjects were training at the same relative load the training protocol induced very different metabolic responses. The reasoning for this large variation in response is still not clear. The current researchers speculate that differences in muscle fiber composition may partially explain the variation in responses seen. Lactate responses have been used previously to set training intensity zones for endurance training and team sports training as well as monitoring fatigue levels (Billat, 1996; Eniseler, 2005; Faude, Kindermann, \& Meyer, 2009; Halson, 2014). It may be more appropriate to utilize an individual's lactate response to prescribe MERT.

The current study observed a similar lactate response following a single session of MERT compared to a single session of HIIT, but did not see similar adaptations even though a similar number of training sessions over a similar length of time was utilized(Burgomaster et al., 2006; Burgomaster et al., 2005; Edge, Bishop, et al., 2006; Jakeman et al., 2012; Laursen et al., 2005; B. Rønnestad et al., 2014). This divergence in the time course of adaptations for the two training methodologies is interesting and remains unclear at the moment. However, it can be speculated the differences in the number of muscular contractions performed during a single session and over the course of the training protocol maybe a reason for differences. A single session of MERT would require between 108 and 135 muscular 
contractions. Examining the HIIT protocol used by Burgomaster et al. (2006), 4 to 730 second sprints, an individual maintaining a cadence between 90 and 100 revolutions per minute could potentially perform up to 315 to 350 muscular contractions per session (Burgomaster et al., 2006). There could potentially be differences in the length of time for exercise between the two protocols. Future studies aiming to immolate the response and adaptions of lactate kinetics associated with HIIT using MERT should take these differences into consideration when designing a training program. Eight sessions of MERT over four weeks was chosen to not only immolate the training program length used in several HIIT studies, but to also coincide with the typical length of a single mesocycle (Baechle \& Earle, 2008; Burgomaster et al., 2006; Burgomaster et al., 2005; Jakeman et al., 2012; Laursen et al., 2005; B. Rønnestad et al., 2014). The optimal length of time and the proper implementation of MERT into a macrocycle still needs further research. The current research indicates that at least 2 mesocycles (16 sessions over 8 weeks) is needed for adaptions in lactate kinetics using MERT.

Although the aerobic training of subjects was not directly monitored by the researchers, as it was performed outside the laboratory setting; the researchers do not believe that this aspect of the study negates any of the findings of the current study and provides for a real world application. Additionally, subjects were contacted bi-weekly to ensure that the subjects maintained similar diet and eating habits as well maintaining aerobic training volume within the previously described ranges. The aerobic training was not prescribed as it would have posed a difficult task due to the multitude of aerobic activities the subjects participated in. Also, the goal of the study was to examine the adaptations of the additional resistance training to the current aerobic training of the participants. A similar experimental design was utilized by Hickson et al. (1988) and recently by Karsten et al. (2016), Lantis et al. (2017) (2017), and Farrell et al. (2017) where all strength training was performed under the supervision of the researchers while all aerobic training was performed independently outside the lab when evaluating the potential for simultaneous strength and endurance training to improve endurance performance (Farrell III et al., 2017; Hickson, Dvorak, Gorostiaga, Kurowski, \& Foster, 1988; Karsten et al., 2016; Lantis et al., 2017). The current study is not without limitations. All laboratory visits were scheduled for the same time each visit to control for fluctuations in fatigue and nutrient timing. Subjects were instructed to abstain from exercise at least $12 \mathrm{hrs}$ prior to each visit to the laboratory. Subjects were instructed to maintain their current dietary regiment. Diet was not controlled or monitored in the current study and could be considered a limitation. The current study had a small sample size and was thus statistically underpowered, increasing the risk for Type II error. Future studies should aim to have a larger sample size and potentially implant an athlete tracking system to ensure athlete compliance with external training (GPS and/or heart rate monitoring)

\section{CONCLUSION}

The current study observed that MERT does indeed induce a lactate accumulation similar to that seen in HIIT, as previously speculated. (Edge, Bishop, et al., 2006; Edge, Hill-Haas, et al., 2006). Although a similar lactate response was observed a similar time course in adaptations for improved lactate kinetics was not observed. Previous research utilizing HIIT and SIT have shown improvements in lactate kinetics and aerobic power in as few as 4 to 8 sessions (Beyranvand, 2017; Burgomaster et al., 2006; Burgomaster et al., 2005; Jakeman et al., 2012; Laursen et al., 2005; B. Rønnestad et al., 2014; Talanian et al., 2007). The current research study found that 4 weeks (8 sessions) of MERT did not provide a sufficient stimulus to result in cardiorespiratory and metabolic adaptions to improve aerobic exercise performance. No improvements in $\mathrm{VO}_{2} \max$, GET, or OBLA were observed in the EXP group. However, the EXP group did show a significant improvement in LC and LE 1RM strength. The current study is one of the first to observe lactate kinetics throughout a MERT program. A varied response to the same relative stimulus was observed during the training. Further research is needed to understand the varied responses and how to individualize and prescribe MERT sessions based upon lactate responses. Further research is also needed to understand an optimal length for MERT as the current protocol does not appear sufficient enough to see improvements in aerobic performance.

\section{ACKNOWLEDGMENTS}

No external funding was used to support the current study. The authors have no relationships with any companies that who will benefit from the results of the present study.

\section{REFERENCES}

Aagaard, P., Andersen, J., Bennekou, M., Larsson, B., Olesen, J., Crameri, R., Kjaer, M. (2011). Effects of resistance training on endurance capacity and muscle fiber composition in young top-level cyclists. Scandinavian journal of medicine \& science in sports, 21(6), 298-307.

Baechle, T. R., \& Earle, R. W. (2008). Essentials of strength training and conditioning: Human kinetics.

Beaver, W. L., Wasserman, K., \& Whipp, B. J. (1986). A new method for detecting anaerobic threshold by gas exchange. Journal of applied physiology, 60(6), 2020-2027.

Bergman, B. C., Wolfel, E. E., Butterfield, G. E., Lopaschuk, G. D., Casazza, G. A., Horning, M. A., \& Brooks, G. A. (1999). Active muscle and whole body lactate kinetics after endurance training in men. Journal of applied physiology, 87(5), 1684-1696.

Beyranvand, F. (2017). Sprint Interval Training Improves Aerobic and Anaerobic Power in Trained Female Futsal Players. International Journal of Kinesiology and Sports Science, 5(2), 43-47.

Billat, L. V. (1996). Use of blood lactate measurements for prediction of exercise performance and for control of training. Sports Medicine, 22(3), 157-175. 
Borg, G. (1970). Perceived exertion as an indicator of somatic stress. Scand J Rehabil Med, 2, 92-98.

Brooks, G. A. (2010). What does glycolysis make and why is it important? Journal of applied physiology, 108(6), 1450-1451.

Burgomaster, K. A., Heigenhauser, G. J., \& Gibala, M. J. (2006). Effect of short-term sprint interval training on human skeletal muscle carbohydrate metabolism during exercise and time-trial performance. Journal of applied physiology, 100(6), 2041-2047.

Burgomaster, K. A., Hughes, S. C., Heigenhauser, G. J., Bradwell, S. N., \& Gibala, M. J. (2005). Six sessions of sprint interval training increases muscle oxidative potential and cycle endurance capacity in humans. Journal of applied physiology, 98(6), 1985-1990.

Campos, G. E., Luecke, T. J., Wendeln, H. K., Toma, K., Hagerman, F. C., Murray, T. F., Staron, R. S. (2002). Muscular adaptations in response to three different resistance-training regimens: specificity of repetition maximum training zones. European journal of applied physiology, 88(1-2), 50-60.

Cohen, J. (1988). Statistical power analysis for the behavioral sciences ( $2^{\text {nd }} e d$.). Hillsdale, NJ: Erlbaum.

Cruz, R. S. d. O., de Aguiar, R. A., Turnes, T., Penteado Dos Santos, R., Fernandes Mendes de Oliveira, M., \& Caputo, F. (2012). Intracellular shuttle: the lactate aerobic metabolism. The Scientific World Journal, 2012.

Donovan, C. M., \& Brooks, G. A. (1983). Endurance training affects lactate clearance, not lactate production. American Journal of Physiology-Endocrinology And Metabolism, 244(1), E83-E92.

Edge, J., Bishop, D., \& Goodman, C. (2006). The effects of training intensity on muscle buffer capacity in females. European journal of applied physiology, 96(1), 97-105.

Edge, J., Hill-Haas, S., Goodman, C., \& Bishop, D. (2006). Effects of Resistance Training on $\mathrm{H}^{\wedge}+$ Regulation, Buffer Capacity, and Repeated Sprints. Medicine and Science in Sports and Exercise, 38(11), 2004.

Eniseler, N. (2005). Heart rate and blood lactate concentrations as predictors of physiological load on elite soccer players during various soccer training activities. The Journal of Strength \& Conditioning Research, 19(4), 799-804.

Farrell III, J. W., Lantis, D. J., Ade, C. J., Cantrell, G. S., \& Larson, R. D. (2017). Aerobic exercise supplemented with muscular endurance training improves onset of blood lactate accumulation. The Journal of Strength \& Conditioning Research, (in press).

Faude, O., Kindermann, W., \& Meyer, T. (2009). Lactate threshold concepts. Sports Medicine, 39(6), 469-490.

Figueira, T. R., Caputo, F., Pelarigo, J. G., \& Denadai, B. S. (2008). Influence of exercise mode and maximal lactatesteady-state concentration on the validity of OBLA to predict maximal lactate-steady-state in active individuals. Journal of Science and Medicine in Sport, 11(3), 280-286.

Fleck, S. J. (1994). Detraining: Its Effects on Endurance and Strength. Strength \& Conditioning Journal, 16(1), 22-28.
Halson, S. L. (2014). Monitoring training load to understand fatigue in athletes. Sports Medicine, 44(2), 139-147.

Heyward, V. H. (1992). Advanced Fitness Assessment and Exercise Prescription. Medicine \& Science in Sports \& Exercise, 24(2), 278.

Hickson, R., Dvorak, B., Gorostiaga, E., Kurowski, T., \& Foster, C. (1988). Potential for strength and endurance training to amplify endurance performance. Journal of applied physiology, 65(5), 2285-2290.

Jakeman, J., Adamson, S., \& Babraj, J. (2012). Extremely short duration high-intensity training substantially improves endurance performance in triathletes. Applied Physiology, Nutrition, and Metabolism, 37(5), 976-981.

Jung, A. P. (2003). The impact of resistance training on distance running performance. Sports Medicine, 33(7), 539-552.

Karsten, B., Stevens, L., Colpus, M., Larumbe-Zabala, E., \& Naclerio, F. (2016). The effects of a sports specific maximal strength and conditioning training on critical velocity, anaerobic running distance and $5-\mathrm{km}$ race performance. International journal of sports physiology and performance, $11(1), 80-85$.

Kraemer, W., Adams, K., Cafarelli, E., Dudley, G., Dooly, C., Feigenbaum, M., Hoffman, J. (2002). J. Potteiger, MH Stone, NA Ratamess, and T. Triplett-Mcbride. American College of Sports Medicine position stand. Progression models in resistance training for healthy adults. Medicine \& Science in Sports \& Exercise, 34(2), 364-380.

Lantis, D. J., Farrell III, J. W., Cantrell, G. S., \& Larson, R. D. (2017). Eight Weeks of High-Volume Resistance Training Improves Onset of Blood Lactate in Trained Individuals. The Journal of Strength \& Conditioning Research, 31(8), 2176-2182.

Laursen, P. B., \& Jenkins, D. G. (2002). The scientific basis for high-intensity interval training. Sports Medicine, 32(1), 53-73.

Laursen, P. B., Shing, C. M., Peake, J. M., Coombes, J. S., \& Jenkins, D. G. (2005). Influence of high-intensity interval training on adaptations in well-trained cyclists. Journal of Strength and Conditioning Research, 19(3), 527.

Losnegard, T., Mikkelsen, K., Rønnestad, B., Hallén, J., Rud, B., \& Raastad, T. (2011). The effect of heavy strength training on muscle mass and physical performance in elite cross country skiers. Scandinavian journal of medicine \& science in sports, 21(3), 389-401.

Mikkola, J., Vesterinen, V., Taipale, R., Capostagno, B., Häkkinen, K., \& Nummela, A. (2011). Effect of resistance training regimens on treadmill running and neuromuscular performance in recreational endurance runners. Journal of Sports Sciences, 29(13), 1359-1371.

Millet, G. P., Jaouen, B., Borrani, F., \& Candau, R. (2002). Effects of concurrent endurance and strength training on running economy and $\mathrm{VO} \sim 2$ kinetics. Medicine and Science in Sports and Exercise, 34(8), 1351-1359.

Rogatzki, M. J., Wright, G. A., Mikat, R. P., \& Brice, A. G. (2014). Blood Ammonium and Lactate Accumulation Response to Different Training Protocols Using the Parallel Squat Exercise. The Journal of Strength \& Conditioning Research, 28(4), 1113-1118. 
Rønnestad, B., Hansen, J., \& Ellefsen, S. (2014). Block periodization of high-intensity aerobic intervals provides superior training effects in trained cyclists. Scandinavian journal of medicine \& science in sports, 24(1), 34-42.

Rønnestad, B. R., Hansen, E. A., \& Raastad, T. (2010). Effect of heavy strength training on thigh muscle cross-sectional area, performance determinants, and performance in well-trained cyclists. European journal of applied physiology, 108(5), 965-975.

Rønnestad, B. R., Kojedal, Ø., Losnegard, T., Kvamme, B., \& Raastad, T. (2012). Effect of heavy strength training on muscle thickness, strength, jump performance, and endurance performance in well-trained Nordic Combined athletes. European journal of applied physiology, 112(6), 2341-2352.

Schott, J., McCully, K., \& Rutherford, O. (1995). The role of metabolites in strength training. European journal of applied physiology and occupational physiology, 71(4), 337-341.
Sjödin, B., \& Jacobs, I. (1981). Onset of blood lactate accumulation and marathon running performance. International journal of sports medicine, 2(1), 23-26.

Sjödin, B., Jacobs, I., \& Svedenhag, J. (1982). Changes in onset of blood lactate accumulation (OBLA) and muscle enzymes after training at OBLA. European journal of applied physiology and occupational physiology, 49(1), 45-57.

Talanian, J. L., Galloway, S. D., Heigenhauser, G. J., Bonen, A., \& Spriet, L. L. (2007). Two weeks of high-intensity aerobic interval training increases the capacity for fat oxidation during exercise in women. Journal of applied physiology, 102(4), 1439-1447.

Tanaka, H., \& Swensen, T. (1998). Impact of resistance training on endurance performance. Sports Medicine, 25(3), 191-200.

Zupan, M. F., \& Petosa, P. S. (1995). Aerobic and Resistance Cross-Training for Peak Triathlon Performance. Strength \& Conditioning Journal, 17(5), 7-12. 